\title{
Effect of the Kondo correlation on Shot Noise in a Quantum Dot
}

\author{
Bing Dong and X. L. Lei \\ Department of Physics, Shanghai Jiaotong University, 1954 Huashan Road, Shanghai 200030, P. R. China
}

\begin{abstract}
The current noise in a quantum dot coupled to two leads is investigated in the Kondo regime with and without the influence of magnetic fields by employing a finite- $U$ slave-boson mean field approach to calculate the current-current correlation function at zero temperature. The numerical results show that the Fano factor is always reduced significantly due to the Kondo-correlation effect, and the most pronounced suppression appears at the electron-hole symmetry case $2 \epsilon_{d}+U=0$. In addition, the application of a magnetic field enhance the Fano factor at small external voltage, which is attributed to the reduction of the Kondo-enhanced density-of-state and transmission probability in a quantum dot.

PACS numbers: 72.10.Fk, 72.15.Qm, 72.70.+m, 73.50.Fq
\end{abstract}

\section{INTRODUCTION}

Since the great breakthroughs in experiments were made several years ago, in which a semiconductor quantum dot (QD) device was subtly designed by using nano-electronics technique and the conductance through this QD was measured to show the well-known Kondo effects, 每 the study of electronic transport through QD has become current interesting subject. 15 So far, the main features of the Kondo effect that have been explored in QD are Kondo-assisted enhancement of conductance, its specific temperature dependence, a peak splitting in a magnetic field, zero-bias maximum of differential conductance in the Kondo regime, and integer-spin Kondo effect.

Electrical current through a conductor is always fluctuation with time and manifests the consequence of the quantization of the charge carriers, which is usually refered to as the shot noise in literature 16 The noise characteristic of a conductor is important and interesting because the spectrum of shot noise contains some information related to electron transport in the conductor, which can not be obtained only through measuring the conductance. For example, shot noise experiments can determine the kinetics of electron, and reveal information on the correlatiops of electronic wave function. Therefore, shot noise has focused considerable investigation in mesoscopic systems.17 28 It is known that the zero-frequency shot noise $S(0)$ for a classical conductor is characterized by the Poisson value $S_{P}(0)=2 e\langle I\rangle$ ( $\langle I\rangle$ is the average current), while the shot noise in a non-interacting mesoscopic conductor is always reduced by the Pauli exclusion in comparison with the Poisson value. Of course, shot noise is also influenced by electron-electron interaction. However, it seems that, depending on the details of the systems under investigation, both suppression 18.19 and enhancement 2021 of shot noise from the classical value due to Coulomb interaction are observed by several experiments.

Up to now, most of the theoretical studies on shot noise in mesoscopic systems are concentrated on the noninteracting electrons. To our knowledge, only a few papers deal with the shot noise of QD in the strongly correlated Kondo regime. Hershfield 26 computed perturbatively the zero-frequency current noise in the Hartree approximation based on the Green's function (GF) approach. He found the interaction can either enhance or reduce the shot noise. Yamaguchi and Kawamura27 performed a complementary analysis by treating the tunneling Hamiltonian perturbatively and revealed a large suppression as compared with the Poisson value due to the on-site Coulomb interaction. However both of their results are valid for the high-temperature regime and do not describe the Kondo physics because of their perturbation scheme. Ding and $\mathrm{Ng} 2 \mathrm{~s}$ calculated the zero-frequency and frequency-dependent shot noise for the Kondo regime at low temperature $T=T_{K}$ ( $T_{K}$ denotes the Kondo temperature) by employing equation-of-motion method and Ng's ansatz for the correlation GF. Their results also demonstrated suppression of the shot noise below the non-interacting value for any applied voltage. They evaluated the retarded GF of the current operator and the occupation number operator instead of the current-current correlation GF, which must be treated for investigation of shot noise by means of nonequilibrium GF approach. Then shot noise problems in strongly correlated systems remain not to be solved.

Mgre recently, a new slave-boson mean field (SBMF) approach has been developed to investigate transport through QD29 with arbitrary strength of the Coulomb interaction, which is an extension of the saddle-point approximation to the auxiliary-boson functional integral method for the Anderson models, suggested by Kotliar and Ruckenstein, 30 to the nonequilibrium situations. It has been confirmed that this formulation's simplest saddle-point approximation for all introduced Bose fields and Lagrange multipliers is, at zero temperature, equivalent to the results derived from the Gutzwiller variational wave function, 30 the well-known analytical approach for strongly correlated fermions. It 
is believed that since more auxiliary parameters, which have unambiguous physical meanings, are introduced in this scheme than in the usual slaueboson formulation, this new SBMFT provides more precise description for the Kondotype transport through QD.29 The purpose of the present paper is to further generalize this finite- $U$ approach to investigate the nonlinear shot noise of QD with and without the influence of magnetic fields.

We arrange the rest parts of the paper as following. In the second section we briefly discuss the equivalent slaveboson field Hamiltonian and self-consistent equations to determine the unknown expectation values for slave-boson operators within the SBMF approach. Meanwhile, we define the "average" noise $S(\omega)$ and the noise $S_{\alpha}(\omega)$ of the lead $\alpha$ and directly give their expressions. At zero temperature, we find that the "average" zero-frequency shot noise coincides with the zero-frequency shot noise for any lead $\alpha$, but this fact is not always satisfied in general condition. The numerical calculation and discussion are performed in the following section. Fano factor which characterizes the deviation of the shot noise from the classical result is obtained and its limit value at low applied voltage is analysed. The concrete resutls clearly demonstrate that the strong on-site Coulomb interaction can significantly reduce the Fano factors of QD in the Kondo regime, thus largely suppress the shot noise below the Poisson value. On the other hand, application of magnetic fields enhances the Fano factor evidently, which can be understood by the fact that the Kondo induced enhancement of the transmission probability through QD is weakened under the influence of magnetic fields. As our knowledge, this is the first time in literature to reveal the effect of magnetic fields on the shot noise in the strongly correlated QD. Finally, a conclusion is given in Section 5.

\section{FINITE- $U$ SLAVE-BOSON MEAN FIELD APPROACH AND NONLINEAR SHOT NOISE FORMULA}

Transport through a QD coupled to two reservoirs in the presence of external voltages and of magnetic fields can be described by the Anderson single impurity model:

$$
H=\sum_{\sigma k \alpha} \epsilon_{k \alpha \sigma}^{\prime} c_{k \alpha \sigma}^{\dagger} c_{k \alpha \sigma}+\sum_{\sigma} \epsilon_{d \sigma} c_{d \sigma}^{\dagger} c_{d \sigma}+U n_{d \uparrow} n_{d \downarrow}+\sum_{\sigma k \alpha}\left(V_{\alpha} c_{k \alpha \sigma}^{\dagger} c_{d \sigma}+\text { H.c. }\right),
$$

where $\epsilon_{k \alpha \sigma}^{\prime}=\epsilon_{k \alpha \sigma}+V_{\alpha}$ represents the conduction electron energy under the application of the external voltage $V_{\alpha}$ to

the lead $\alpha \cdot c_{k \alpha \sigma}^{\dagger}\left(c_{k \alpha \sigma}\right)$ are the creation (annihilation) operators for electrons in the lead $\alpha(=\mathrm{L}, \mathrm{R})$. When a total external voltage $V$ is applied between the two leads, their chemical potential difference is $\mu_{\mathrm{L}}-\mu_{\mathrm{R}}=e V$. The two leads are assumed to be in local equilibrium and their distribution functions are given by the Fermi distribution functions $f_{\alpha}(\omega)=\left[1+\exp \left(\omega-\mu_{\alpha}\right) / k_{\mathrm{B}} T\right]^{-1}(\alpha=\mathrm{L}$ or $\mathrm{R})$. This assumption is practically correct because the two reservoirs respond to an applied field much faster than the center region, i.e., the QD. Under the influence of a magnetic field $B$, the discrete energy levels $\epsilon_{d}$ in the QD are split into $\epsilon_{d \sigma} \equiv \epsilon_{d}+\sigma h(\sigma= \pm 1)$ for up and down spins, where $2 h=g \mu_{\mathrm{B}} B$ ( $g$ is the Landé factor and $\mu_{\mathrm{B}}$ the Bohr magneton) is the Zeeman energy. The other parameters $U$, and $V_{\alpha}$ stand for the Coulomb interaction, and the coupling between the QD and the reservoir $\alpha$, respectively. According to the KR slave-boson representation 30 we introduce four auxiliary Bose fields $e, p_{\sigma}(\sigma= \pm 1)$, and $d$, which act respectively as projection operators onto the empty, singly occupied (with spin up and down), and doubly occupied electronic states at the QD. In order to eliminate additional unphysical states, three constraints have to impose on these bosons

$$
\begin{aligned}
& \sum_{\sigma} p_{\sigma}^{\dagger} p_{\sigma}+e^{\dagger} e+d^{\dagger} d=1, \\
& c_{d \sigma}^{\dagger} c_{d \sigma}=p_{\sigma}^{\dagger} p_{\sigma}+d^{\dagger} d, \quad \sigma= \pm 1 .
\end{aligned}
$$

Equations (2) and (3) are the completeness relation and the condition for the correspondence between fermions and bosons, respectively. In the physical subspace defined by these constraints, the fermion operators $c_{d \sigma}^{\dagger}$ and $c_{d \sigma}$ of the QD in the hopping terms are replaced by

$$
z_{\sigma}^{\dagger} c_{d \sigma}^{\dagger}, \quad c_{d \sigma} z_{\sigma},
$$

so that the matrix elements are the same in the combined fermion-boson Hilbert space as those in the original one Eq. (1). Here

$$
z_{\sigma}=\left(1-d^{\dagger} d-p_{\sigma}^{\dagger} p_{\sigma}\right)^{-1 / 2}\left(e^{\dagger} p_{\sigma}+p_{\bar{\sigma}}^{\dagger} d\right)\left(1-e^{\dagger} e-p_{\bar{\sigma}}^{\dagger} p_{\bar{\sigma}}\right)^{-1 / 2} .
$$

Therefore, the Hamiltonian (11) can be replaced by the following effective Hamiltonian in terms of auxiliary boson operators and of decorated fermion operators: 


$$
\begin{aligned}
H_{\mathrm{eff}}= & \sum_{k \alpha \sigma} \epsilon_{k \alpha \sigma}^{\prime} c_{k \alpha \sigma}^{\dagger} c_{k \alpha \sigma}+\sum_{\sigma} \epsilon_{d \sigma} c_{d \sigma}^{\dagger} c_{d \sigma}+U d^{\dagger} d+\sum_{k \alpha \sigma}\left(V_{\alpha} c_{k \alpha \sigma}^{\dagger} c_{d \sigma} z_{\sigma}+\text { H.c. }\right) \\
& +\lambda^{(1)}\left(\sum_{\sigma} p_{\sigma}^{\dagger} p_{\sigma}+e^{\dagger} e+d^{\dagger} d-1\right)+\sum_{\sigma} \lambda_{\sigma}^{(2)}\left(c_{d \sigma}^{\dagger} c_{d \sigma}-p_{\sigma}^{\dagger} p_{\sigma}-d^{\dagger} d\right) .
\end{aligned}
$$

The constraints are incorporated via the three Lagrange multipliers, $\lambda^{(1)}$ and $\lambda_{\sigma}^{(2)}$. Under the framework of SBMF approach, the four slave Bose fields can be assumed as c-numbers and replaced by their corresponding expectation values. It will be seen in the following that, under this approximation, transport through the QD can be characterized by the seven factitious parameters $e, p_{\sigma}, d, \lambda^{(1)}$, and $\lambda_{\sigma}^{(2)}$.

Since the Hamiltonian (6) describing the leads is noninteracting, the unperturbed retarded (advanced) GFs $g_{k \alpha \sigma}^{r, a}\left(t, t^{\prime}\right)$ and "lesser" ("greater") GFs $g_{k \alpha \sigma}^{<,>}\left(t, t^{\prime}\right)$ for the lead $\alpha$ are

$$
\begin{aligned}
& g_{k \alpha \sigma}^{r(a)}\left(t, t^{\prime}\right) \equiv \mp i \theta\left( \pm t \mp t^{\prime}\right)\left\langle\left\{c_{k \alpha \sigma}^{\dagger}\left(t^{\prime}\right), c_{k \alpha \sigma}(t)\right\}\right\rangle=\mp i \theta\left( \pm t \mp t^{\prime}\right) e^{-i \epsilon_{k \alpha \sigma}\left(t-t^{\prime}\right)} \\
& g_{k \alpha \sigma}^{<}\left(t, t^{\prime}\right) \equiv i\left\langle c_{k \alpha \sigma}^{\dagger}\left(t^{\prime}\right) c_{k \alpha \sigma}(t)\right\rangle=i f_{\alpha}\left(\epsilon_{k \alpha \sigma}\right) e^{-i \epsilon_{k \alpha \sigma}\left(t-t^{\prime}\right)} \\
& g_{k \alpha \sigma}^{>}\left(t, t^{\prime}\right) \equiv-i\left\langle c_{k \alpha \sigma}(t) c_{k \alpha \sigma}^{\dagger}\left(t^{\prime}\right)\right\rangle=-i\left[1-f_{\alpha}\left(\epsilon_{k \alpha \sigma}\right)\right] e^{-i \epsilon_{k \alpha \sigma}\left(t-t^{\prime}\right)} .
\end{aligned}
$$

However for the transport problems concerned in this paper, the electrons in the QD are in a nonequilibrium state, to be determined by their coupling to the two leads and to the applied voltage. In order to describe the nonequilibrium state of electrons, we define the retarded (advanced) and lesser (greater) GFs for the QD as follows: $G_{d \sigma}^{r(a)}\left(t, t^{\prime}\right) \equiv$ $\pm i \theta\left( \pm t \mp t^{\prime}\right)\left\langle\left\{c_{d \sigma}(t), c_{d \sigma}^{\dagger}\left(t^{\prime}\right)\right\}\right\rangle, G_{d \sigma}^{<}\left(t, t^{\prime}\right) \equiv i\left\langle c_{d \sigma}^{\dagger}\left(t^{\prime}\right) c_{d \sigma}(t)\right\rangle$ and $G_{d \sigma}^{<}\left(t, t^{\prime}\right) \equiv-i\left\langle c_{d \sigma}(t) c_{d \sigma}^{\dagger}\left(t^{\prime}\right)\right\rangle$. It is clear that for the effective Hamiltonian (6) the Fourier transforms $G_{d \sigma}^{r, a,<}(\omega)$ of these GFs can be readily given, in the wide-band limit, as

$$
\begin{aligned}
G_{d \sigma}^{r(a)}(\omega) & =\frac{1}{\omega-\tilde{\epsilon}_{d \sigma} \pm i \tilde{\Gamma}_{\sigma}} \\
G_{d \sigma}^{<}(\omega) & =\frac{i \tilde{\Gamma}_{\sigma}\left[f_{L}(\omega)+f_{R}(\omega)\right]}{\left(\omega-\tilde{\epsilon}_{d \sigma}\right)^{2}+\tilde{\Gamma}_{\sigma}^{2}}
\end{aligned}
$$

where $\tilde{\Gamma}_{\sigma}=\left(\Gamma_{L}+\Gamma_{R}\right)\left|z_{\sigma}\right|^{2} / 2$ with $\Gamma_{\alpha}=2 \pi \sum_{k \alpha}\left|V_{\alpha}\right|^{2} \delta\left(\omega-\epsilon_{k \alpha \sigma}\right)$ being the coupling strength between the QD level and the lead $\alpha$. Note that these formula are similar with those for noninteracting electrons, except with the effective energy level $\tilde{\epsilon}_{d \sigma}=\epsilon_{d \sigma}+\lambda_{\sigma}^{(2)}$ and the effective coupling constant $\tilde{\Gamma}_{\sigma}$ instead, which renormalize the GFs of the QD due to the strong Coulomb repulsion under the approximation employed here. In the present paper, we focus our attention on the symmetric systems $\Gamma_{L}=\Gamma_{R}=\Gamma$ and take the coupling strength $\Gamma$ as the energy unit throughout the paper. Moreover, the greater GF can be easily obtained from the relationship $G^{r}-G^{a}=G^{>}-G^{<}$. Besides, some other GFs are used in the following derivation of electric current and shot noise spectral. So we definite and treat them in advance. For example, we define the lesser GFs: $G_{d \sigma, k \alpha \sigma}^{<}\left(t, t^{\prime}\right) \equiv i\left\langle c_{k \alpha \sigma}^{\dagger}\left(t^{\prime}\right) c_{d \sigma}(t)\right\rangle, G_{k \alpha \sigma, d \sigma}^{<}\left(t, t^{\prime}\right) \equiv i\left\langle c_{d \sigma}^{\dagger}\left(t^{\prime}\right) c_{k \alpha \sigma}(t)\right\rangle$, and $G_{k \alpha \sigma, k^{\prime} \beta \sigma}^{<}\left(t, t^{\prime}\right) \equiv i\left\langle c_{k^{\prime} \beta \sigma}^{\dagger}\left(t^{\prime}\right) c_{k \alpha \sigma}(t)\right\rangle$ and of course their corresponding retarded, advanced, and greater GFs. With the effective Hamiltonian (6), these GFs can be readily related with the GFs of the QD by applying the Langreth analytic continuation rules 31 :

$$
\begin{aligned}
G_{d \sigma, k \alpha \sigma}^{<}\left(t, t^{\prime}\right) & =\int d t_{1} V_{\alpha}^{*} z_{\sigma}^{*}\left[G_{d \sigma}^{<}\left(t, t_{1}\right) g_{k \alpha \sigma}^{a}\left(t_{1}, t^{\prime}\right)+G_{d \sigma}^{r}\left(t, t_{1}\right) g_{k \alpha \sigma}^{<}\left(t_{1}, t^{\prime}\right)\right], \\
G_{k \alpha \sigma, d \sigma}^{<}\left(t, t^{\prime}\right) & =\int d t_{1} V_{\alpha} z_{\sigma}\left[g_{k \alpha \sigma}^{<}\left(t, t_{1}\right) G_{d \sigma}^{a}\left(t_{1}, t^{\prime}\right)+g_{k \alpha \sigma}^{r}\left(t, t_{1}\right) G_{d \sigma}^{<}\left(t_{1}, t^{\prime}\right)\right], \\
G_{k \alpha \sigma, k^{\prime} \beta \sigma}^{<}\left(t, t^{\prime}\right) & =g_{k \alpha \sigma}^{<}\left(t, t^{\prime}\right) \delta_{k k^{\prime}} \delta_{\alpha \beta}+\int d t_{1} V_{\beta} z_{\sigma}\left[g_{k \alpha \sigma}^{<}\left(t, t_{1}\right) G_{d \sigma, k^{\prime} \beta \sigma}^{a}\left(t_{1}, t^{\prime}\right)+g_{k \alpha \sigma}^{r}\left(t, t_{1}\right) G_{d \sigma, k^{\prime} \beta \sigma}^{<}\left(t_{1}, t^{\prime}\right)\right] .
\end{aligned}
$$

The current operator flowing from the lead $\alpha$ to the QD can be evaluated from the time evolution of the occupied number operator of the lead

$$
\begin{aligned}
\hat{I}_{\alpha}(t) & =-\frac{e}{\hbar}\left\langle\frac{d \hat{N}_{\alpha}}{d t}\right\rangle=-i \frac{e}{\hbar}\left[H_{\mathrm{eff}}, \sum_{k \alpha \sigma} c_{k \alpha \sigma}^{\dagger}(t) c_{k \alpha \sigma}(t)\right] \\
& =i \frac{e}{\hbar} \sum_{k \alpha}\left[V_{\alpha} z_{\sigma} c_{k \alpha \sigma}^{\dagger}(t) c_{d \sigma}(t)-V_{\alpha}^{*} z_{\sigma}^{*} c_{d \sigma}^{\dagger}(t) c_{k \alpha \sigma}(t)\right]
\end{aligned}
$$


Because the electric current fluctuates, the currents flowing into QD and flowing out of QD are not in balance. The terminal current is given by the average current $\hat{I}=\left(\hat{I}_{L}-\hat{I}_{R}\right) / 2$. Its statistical expectation yields the current through QD we are interested in. By the help of the Dyson equations (12) and (13), the current formula through the QD takes the form 29

$$
I=\frac{\mathrm{e}}{\hbar} \sum_{\sigma} \int d \omega \tilde{\Gamma}_{\sigma}\left\{f_{L}(\omega)-f_{R}(\omega)\right\} \rho_{\sigma}(\omega)
$$

where $\rho_{\sigma}=-(1 / \pi) \operatorname{Im} G_{d \sigma}^{r}(\omega)$ is the spectral density-of-state (DOS) of the electron in the QD. Starting from the constraints (2), (3) and the equation of motion of the slave-boson operators from the Hamiltonian (6), we can yield the following self-consistent set of equations within the SBMF approach as $(\sigma= \pm 1): 29$

$$
\begin{aligned}
& \sum_{\sigma}\left|p_{\sigma}\right|^{2}+|e|^{2}+|d|^{2}=1 \\
& \frac{1}{2 \pi i} \int d \omega G_{d \sigma}^{<}(\omega)=\left|p_{\sigma}\right|^{2}+|d|^{2} \\
& \frac{1}{2 \pi i} \sum_{\sigma} \frac{\partial \ln z_{\sigma}}{\partial e} \int d \omega G_{d \sigma}^{<}(\omega)\left(\omega-\tilde{\epsilon}_{d \sigma}\right)+2 \lambda^{(1)} e=0 \\
& \frac{1}{2 \pi i} \sum_{\sigma^{\prime}}\left(\frac{\partial \ln z_{\sigma^{\prime}}}{\partial p_{\sigma}^{\dagger}}+\frac{\partial \ln z_{\sigma^{\prime}}}{\partial p_{\sigma}}\right) \int d \omega G_{d \sigma^{\prime}}^{<}(\omega)\left(\omega-\tilde{\epsilon}_{d \sigma^{\prime}}\right)+2\left(\lambda^{(1)}-\lambda_{\sigma}^{(2)}\right) p_{\sigma}=0 \\
& \frac{1}{2 \pi i} \sum_{\sigma} \frac{\partial \ln z_{\sigma}}{\partial d} \int d \omega G_{d \sigma}^{<}(\omega)\left(\omega-\tilde{\epsilon}_{d \sigma}\right)+2\left(U+\lambda^{(1)}-\sum_{\sigma} \lambda_{\sigma}^{(2)}\right) d=0 .
\end{aligned}
$$

Therefore, these equations (17)-(21) form a closed set of self-consistent equations, which can define the seven parameters, and thus describe linear and nonlinear transport through the QD under finite external voltages and magnetic fields.

The shot noise is resulted from the quantization of the charge and to observe it we have to investigate the nonequilibrium (transport) state of the systems. While, from the point of view of practical experiments, it is the external voltage fluctuations which are actually measured and which eventually are converted to current fluctuations. As a result of this fact, the noise can be taken as a response of current to a small amplitude high-frequency voltage superposed on a dc bias: $V_{\alpha}(t)=V_{\alpha 0}+V_{\alpha 1} \cos (\omega t)$, with $V_{\alpha 0}$ and the $V_{\alpha 1}$ term denote the dc and ac bias, respectively. As well the noise power spectrum turns out to be related to the ac conductance in the presence of this high-frequency voltage $V_{\alpha}(t)$. In the linear transport region $V_{\alpha 0} \rightarrow 0$, this gives rise to the well-known Einstein relation $S(\omega)=4 k T \sigma(\omega)$ $(\sigma(\omega)$ is the small signal ac conductance). Naturally, we can use the Hamiltonian (1) to describe the system under the influence of the time-alternating voltage $V_{\alpha}(t)$ and further calculate transport and noise for the QD. It is obvious that after a transient process the system will be driven to an oscillatory steady state, in which the physics quantities will perform as a sum of a dc part under the influence of $V_{\alpha 0}$ and a small amplitude oscillation part at the single driving frequency $\omega$ (fluctuation part in the present problem), if the high-frequency bias is sufficiently small. For example, the current operator $\hat{I}_{\alpha}(t)$ now consists of both the average current $\left\langle I_{\alpha}(t)\right\rangle$ and a small fluctuation $\delta \hat{I}_{\alpha}(t)$

$$
\begin{aligned}
\hat{I}_{\alpha}(t) & =\left\langle I_{\alpha}(t)\right\rangle+\delta \hat{I}_{\alpha}(t) \\
& =I_{\alpha}+\delta \hat{I}_{\alpha}(t)
\end{aligned}
$$

with

$$
\left\langle\delta \hat{I}_{\alpha}(t)\right\rangle=0
$$

With no reason, an important assumption can be made as follows: those introduced auxiliary Bose fields and the Lagrange multipliers also obey this discipline within SBMF approach:

$$
\begin{aligned}
\hat{\mathcal{O}}(t) & =\mathcal{O}_{0}+\delta \hat{\mathcal{O}}(t), \\
\lambda(t) & =\lambda_{0}+\delta \lambda(t)
\end{aligned}
$$

with

$$
\begin{aligned}
\langle\delta \hat{\mathcal{O}}(t)\rangle & =0 \\
\langle\delta \lambda(t)\rangle & =0
\end{aligned}
$$


where the operator $\hat{\mathcal{O}}$ represents any one of the slave-boson operators $e, p_{\sigma}$, and $d$ and $\mathcal{O}_{0}$ the corresponding expectation value. $\lambda$ denotes the Lagrange multipliers $\lambda^{(1)}$ and $\lambda_{\sigma}^{(2)}$ and $\lambda_{0}$ the constant value. Owing to the sufficiently small amplitudes of these fluctuations of Bose fields, we can ignore their minor contribution and only remain their zero order terms, the expectation values $\mathcal{O}_{0}$ and $\lambda_{0}$, in the calculation for the correlation of the current fluctuation. This is our central presumption in this paper. Discussion about the validity of this approximation is beyond the scope of the present paper and the effects of fluctuation of these Bose fields on transport will be investigated in our future publication. With this consideration, the effective Hamiltonian (6) can still be utilized to depict transport through the QD under the voltage $V_{\alpha}(t)$ but with the constant expectation values $\mathcal{O}_{0}$ and $\lambda_{0}$ instead. It turn out to be that this procedure can provide a considerable precise prescription for kondo-type transport through QD with and without magnetic fields.29 In the following, starting from this Hamiltonian (6), we will investigate the shot noise of QD in the Kondo regime. The approximation of slave-boson mean field deals with the on-site Coulomb interaction as a renormalization hopping factor $z_{\sigma}$ [Eq.(5)] and makes the Hamiltonian for the QD system reduce to non-interacting one will largely simplify the calculation for the current-current correlation function.

The power density $S(\omega)$ of the tunneling current fluctuation is defined as the Fourier transform of the following correlation function:

$$
S\left(t-t^{\prime}\right)=\left\langle\delta \hat{I}(t) \delta \hat{I}\left(t^{\prime}\right)+\delta \hat{I}\left(t^{\prime}\right) \delta \hat{I}(t)\right\rangle .
$$

Note that the current operator involved in the definition is the average current. So we term this noise current as "average" noise. However, in the practical experiments, one has to choose either "L" lead or "R" lead to measure the current and noise spectrum. Therefore, it is necessary to define the noise $S_{\alpha}\left(t-t^{\prime}\right)$ of the lead $\alpha$ :

$$
S_{\alpha}\left(t-t^{\prime}\right)=\left\langle\delta \hat{I}_{\alpha}(t) \delta \hat{I}_{\alpha}\left(t^{\prime}\right)+\delta \hat{I}_{\alpha}\left(t^{\prime}\right) \delta \hat{I}_{\alpha}(t)\right\rangle .
$$

In order to evaluate the noise, we substitute the current operator Eq.(15) into Eqs.(28) and (29) and express these quantum statistical (nonequilibrium) average in terms of the nonequilibrium GFs. After a length but straightforward derivation, we obtain:

$$
\begin{aligned}
S_{\alpha}(\omega)= & -\frac{e^{2}}{\hbar} \sum_{\sigma} \tilde{\Gamma}_{\sigma}^{2} \int \frac{d \omega_{1}}{2 \pi}\left\{\left\{f_{\alpha}\left(\omega_{1}\right)\left[1-f_{\alpha}\left(\omega+\omega_{1}\right)\right]\left[G_{d \sigma}^{r}\left(\omega_{1}\right) G_{d \sigma}^{r}\left(\omega+\omega_{1}\right)+G_{d \sigma}^{a}\left(\omega_{1}\right) G_{d \sigma}^{a}\left(\omega+\omega_{1}\right)\right]\right.\right. \\
& +\left[1-f_{\alpha}\left(\omega+\omega_{1}\right)\right] G_{d \sigma}^{<}\left(\omega_{1}\right)\left[G_{d \sigma}^{r}\left(\omega+\omega_{1}\right)-G_{d \sigma}^{a}\left(\omega+\omega_{1}\right)\right]+f_{\alpha}\left(\omega_{1}\right)\left[G_{d \sigma}^{a}\left(\omega_{1}\right)-G_{d \sigma}^{r}\left(\omega_{1}\right)\right] G_{d \sigma}^{>}\left(\omega+\omega_{1}\right) \\
& \left.\left.-G_{d \sigma}^{<}\left(\omega_{1}\right) G_{d \sigma}^{>}\left(\omega+\omega_{1}\right)+\frac{i}{\tilde{\Gamma}_{\sigma}}\left\{\left[1-f_{\alpha}\left(\omega+\omega_{1}\right)\right] G_{d \sigma}^{<}\left(\omega_{1}\right)-f_{\alpha}(\omega) G_{d \sigma}^{>}\left(\omega+\omega_{1}\right)\right\}\right\}+\{\omega \rightarrow-\omega\}\right\} \\
S(\omega)= & -\frac{e^{2}}{4 \hbar} \sum_{\sigma} \tilde{\Gamma}_{\sigma}^{2} \int \frac{d \omega_{1}}{2 \pi}\left\{\left\{\left[f_{L}\left(\omega_{1}\right)\left[f_{R}\left(\omega+\omega_{1}\right)-f_{L}\left(\omega+\omega_{1}\right)\right]+f_{R}\left(\omega_{1}\right)\left[f_{L}\left(\omega+\omega_{1}\right)-f_{R}\left(\omega+\omega_{1}\right)\right]\right]\right.\right. \\
& \times\left[G_{d \sigma}^{r}\left(\omega_{1}\right) G_{d \sigma}^{r}\left(\omega+\omega_{1}\right)+G_{d \sigma}^{a}\left(\omega_{1}\right) G_{d \sigma}^{a}\left(\omega+\omega_{1}\right)\right] \\
& \left.\left.+\frac{i}{\tilde{\Gamma}_{\sigma}}\left\{\left[2-f_{L}\left(\omega+\omega_{1}\right)-f_{R}\left(\omega+\omega_{1}\right)\right] G_{d \sigma}^{<}\left(\omega_{1}\right)-\left[f_{L}\left(\omega+\omega_{1}\right)+f_{R}\left(\omega+\omega_{1}\right)\right] G_{d \sigma}^{>}\left(\omega_{1}\right)\right\}\right\}+\{\omega \rightarrow-\omega\}\right\} .
\end{aligned}
$$

It is very clear that the average noise spectrum $S(\omega)$ is different from the noise spectrum $S_{\alpha}(\omega)$ of the lead $\alpha$. In the nonequilibrium condition, the difference of the noise spectrum between the $L$ and the $R$ leads is also distinct.

In this paper, we focus our attention on the zero-frequency noise spectrum. Assuming $\omega=0$ in the formula (30) and (31), it is found that the averge zero-frequency noise $S(0)$ happens to be equal to the zero-frequency noise $S_{\alpha}(0)$ of the lead $\alpha$,

$$
\begin{aligned}
S(0)=S_{\alpha}(0)=\frac{e^{2}}{2 \hbar} \sum_{\sigma} \tilde{\Gamma}_{\sigma} \int & \frac{d \omega}{2 \pi}\left\{\left[f_{L}(\omega)-f_{R}(\omega)\right]^{2}\left[G_{d \sigma}^{r}(\omega)-G_{d \sigma}^{a}(\omega)\right]^{2}\right. \\
& \left.+\frac{2 i}{\tilde{\Gamma}_{\sigma}}\left\{f_{L}(\omega)\left[1-f_{R}(\omega)\right]+f_{R}(\omega)\left[1-f_{L}(\omega)\right]\right\}\left[G_{d \sigma}^{r}(\omega)-G_{d \sigma}^{a}(\omega)\right]\right\} .
\end{aligned}
$$

The zero-frequency noise power spectrum formula is exactly the same as that derived from the scattering matrix theory for the non-interacting electron systems, 16.25 except the coupling strength $\Gamma$ and GFs $G_{d \sigma}^{r(a)}(\omega)$ for the QD are replaced with the renormalized ones. It is worth noting that at zero temperature the equilibrium noise spectrum for the case of zero frequency is exactly zero, i.e., only the nonequilibrium or shot noise contributs to the zero-frequency noise power spectrum. 


\section{CALCULATION AND DISCUSSION}

In this section, we numerically investigate the Kondo correlation effect on the zero-frequency noise properties of QD in the presence or absence of magnetic fields. Calculation is carried out only at zero temperature in the present paper. For the sake of simplification, an assumption that a symmetric voltage drop, $\mu_{L}=-\mu_{R}=e V / 2$, through the whole systems is made in our calculation. Then, considering symmetric coupling for two tunnel barriers, the current and the zero-frequency noise spectrum are anti-symmetric under bias reversal.

Thanks to the simple form of the retarded and advanced GFs $G_{d \sigma}^{r(a)}(\omega)(10)$, the integrals in Eqs. (16) and (32) can be done exactly at zero temperature. We have

$$
\begin{aligned}
I & =\frac{e}{h} \sum_{\sigma} \tilde{\Gamma}_{\sigma}\left(\arctan \frac{\phi-\tilde{\epsilon}_{d \sigma}}{\tilde{\Gamma}_{\sigma}}+\arctan \frac{\phi+\tilde{\epsilon}_{d \sigma}}{\tilde{\Gamma}_{\sigma}}\right) \\
S(0) & =\frac{e^{2}}{h} \sum_{\sigma} \tilde{\Gamma}_{\sigma}\left\{\arctan \frac{\phi-\tilde{\epsilon}_{d \sigma}}{\tilde{\Gamma}_{\sigma}}+\arctan \frac{\phi+\tilde{\epsilon}_{d \sigma}}{\tilde{\Gamma}_{\sigma}}-\tilde{\Gamma}_{\sigma}\left[\frac{\phi-\tilde{\epsilon}_{d \sigma}}{\left(\phi-\tilde{\epsilon}_{d \sigma}\right)^{2}+\tilde{\Gamma}_{\sigma}^{2}}+\frac{\phi+\tilde{\epsilon}_{d \sigma}}{\left(\phi+\tilde{\epsilon}_{d \sigma}\right)^{2}+\tilde{\Gamma}_{\sigma}^{2}}\right]\right\},
\end{aligned}
$$

in which $\phi=e V$ is the total voltage drop between the left and right leads. It is clear that zero-temperature shot noise is always suppressed in comparison with the Poisson value $S_{P}=2 e I$. What magnitude of the suppression below the Poissonian limit is one of the aspects of noise in mesoscopic systems which triggered many of the theoretical and experimental works 16 The Fano factor $\gamma$ that is the ratio of the actual shot noise to the Poisson noise provides a useful device to address this sub-Poissonian shot noise,

$$
\gamma=\frac{S(0)}{2 e I}
$$

Before devoting to the numerical analysis of $\gamma$, two limit cases are inspected. First, it is easy to gain that in the limit of enough_arge voltage $V \rightarrow \infty$, the Fano factor $\gamma \rightarrow 0.5$ for the symmetric systems, 24 which has been observed in experiment,20 regardless of whether considering the strong Coulomb interaction or not. Secondly, in the opposite limit when the voltage difference is very small $V \rightarrow 0$, we have

$$
\lim _{V \rightarrow 0} \gamma=\sum_{\sigma} \frac{\tilde{\Gamma}_{\sigma}^{2} \tilde{\epsilon}_{d \sigma}^{2}}{\left(\tilde{\epsilon}_{d \sigma}^{2}+\tilde{\Gamma}_{\sigma}^{2}\right)} / \sum_{\sigma} \frac{\tilde{\Gamma}_{\sigma}^{2}}{\tilde{\epsilon}_{d \sigma}^{2}+\tilde{\Gamma}_{\sigma}^{2}}
$$

In absence of magnetic fields, it reduces to

$$
\lim _{V \rightarrow 0} \gamma=\frac{\tilde{\epsilon}_{d}^{2}}{\tilde{\epsilon}_{d}^{2}+\tilde{\Gamma}^{2}}
$$

In Figs. 1(a) and (b) we plot, respectively, $I-V$ characteristic and the bias voltage-dependent zero-frequency shot noise $S(0)$ for the QD with $U=7$ and several different energy levels in the Kondo regime $\epsilon_{d}=-1,-1.5,-2,-3.5$, and $\epsilon_{d}=-6$. For these parameters, the Kondo temperatures $T_{K}$ are about $0.44,0.23,0.14,0.076$, and 0.44 , respectively [the exact Bethe-ansatz give this dynamic energy scale $T_{K}=U \sqrt{\beta} \exp (-\pi / \beta) / 2 \pi, \beta=-2 U \Gamma / \epsilon_{d}\left(U+\epsilon_{d}\right)$ ]. The corresponding differential conductance $d I / d V$ and zero-frequency differential shot noise $d S(0) / d V$ are also depicted as functions of the bias voltage in Figs.1(c) and (d). It is clear that the zero-frequency shot noise is smaller than the Poisson value $S_{P}$ at the whole range of voltages, showing suppression of shot noise spectrum. A zero-bias maximum behavior in $d I / d V-V$ is found in Fig.1(c), demonstrating the typical Kondo feature for these systems under consideration. While the differential shot noise spectrum exhibits different behavior, nonzero-bias maximum, for all of these systems.

Furthermore, for the sake of comparison, we plot the zero-frequency shot noise spectrum and the differential shot noise for the QD without on-site Coulomb interaction $U=0$ in Fig. 2 and its inset. Evidently we can observe from Fig. 1(b) and Fig. 2, that for these system parameters $\epsilon_{d}=-1,-1.5$, and -2 , the zero-frequency noise power pectrum $S(0)$ in the finite interacting case is significantly smaller than those of the non-interacting systems. In equilibrium, the resonance is reached only for the energy level $\epsilon_{d}$ of the non-interacting QD aligning with the chemical potentials $\mu_{L(R)}$ of the two reservoirs, i.e., $\epsilon_{d}=0$ [we assume $\mu_{L(R)}=0$ in calculation, see Fig. 3(c)]. If the energy level $\epsilon_{d}$ of the $\mathrm{DD}$ is far below the chemical potentials of the two reservoirs the transmission probability $T$ is much less than one,29 which means a large suppression of current through the device. But the strong on-site Coulomb interaction can keep the respmance up to $\epsilon_{d}+U=0$. This is the well-known Kondo effect that has been observed recently for QD in experiments 18 Therefore, in the whole range from $\epsilon_{d}=0$ to $\epsilon_{d}=-U$ the transmission probability through the 
QD remains to be nearly 1. As a consequence, the properties of shot noise are complicated in the presence of strong Coulomb interaction and a universal presentation cannot be summarized in this situation.

A convenient way of addressing how the Coulomb interaction affects the shot noise power spectrum in the Kondo regime is to explore the ratio between the shot noise and the current, i.e., the Fano factor $\gamma$. In Figs. 3, we depict the Fano factor with (a) and without (b) the on-site Coulomb repulsion as a function of the external voltage for the same system parameters as those in Fig. 1. As expected, the Fano factors approach to 0.5 at large voltage for both of the two cases. However, excepting this limit behavior, tremendous difference between the two cases is explicitly observed in the whole range of voltages under consideration. We will interpret these phenomena in a qualitative way as follows.

The classical noise theory yields that the noise $S(0) \propto T(1-T)$, which completely vanishes for a perfectly transparent scatterer $T=1$ and for a perfectly reflecting scatterer $T=0$ and reaches a maximum at $T=1 / 216$ Meanwhile, the Landauer-Büttick scattering theory gives the current to be proportional to the transmission coefficient $I \propto T$. Thus we have $\gamma \propto(1-T)$. In the limit of low-transparency $T \ll 1$ the shot noise $S(0)$ is given by the Poissqn $S_{P}$ and the Fano factor $\gamma$ is equal to 1 . So it is helpful to review the transmission coefficient through QD. 32 , 33 In nonequilibrium condition and under the influence of a magnetic field, the transmission amplitude is related to the retarded $\mathrm{GF} G_{d \sigma}^{r}(\omega)$ of the QD,

$$
t_{d}=\frac{1}{2} \sum_{\sigma} \tilde{\Gamma}_{\sigma} G_{d \sigma}^{r}(\phi),
$$

at zero temperature. Substituting the expression of the retarded GF Eq.(10) into Eq.(38), we derive the transmission coefficient $\left|t_{d}\right|^{2}$ in the linear limit,

$$
\left|t_{d}\right|^{2}=\frac{1}{4} \sin ^{2}\left(\pi n_{\sigma}\right) \sin ^{2}\left(\pi n_{\bar{\sigma}}\right)\left\{4+\left[\cot \left(\pi n_{\sigma}\right)+\cot \left(\pi n_{\bar{\sigma}}\right)\right]^{2}\right\}
$$

where $n_{\sigma}$ denotes the occupation number of electrons with spin $\sigma$ in QD 29

$$
n_{\sigma}=\frac{1}{2}-\frac{1}{\pi} \arctan \left(\frac{\tilde{\epsilon}_{d \sigma}}{\tilde{\Gamma}_{\sigma}}\right) .
$$

In absence of a magnetic field, the transmission coefficient $\left|t_{d}\right|$ Eq.(39) reduces to the formula of the Ref.( 33 ) (Eq.(3) in that paper),

$$
\left|t_{d}\right|^{2}=\sin ^{2}\left(\pi n_{\sigma}\right)
$$

Using Eq.(41), we calculate the transmission coefficient $\left|t_{d}\right|^{2}$ of the QD under several applied external voltages with and without considering the Coulomb interaction, which are plotted as a function of the energy level in Fig. 3(c). It is obvious that for those energy levels, $\left|t_{d}\right|^{2}$ of the non-interacting QD are always smaller than those of the strongly interacting QD in zero voltage limit. Specially, we can easily observe that an approximatively full transparency is established in the vicinity of the electron-hole symmetry $\epsilon_{d}=-U / 2=-3.5$. At this point, the Kondo effect is of most importance and the transmission probability $\left|t_{d}\right|^{2}$ through the QD is nearly equal to 1 . Thus it is rational that the Fano factor demonstrates the most pronounced reduction and an approximate zero value at small voltages, as shown by the dot-dashed line in Fig. 3(a). Therefore it is self-evident from the formula $\gamma \propto 1-\left|t_{d}\right|^{2}$ that the Coulomb interaction suppresses the Fano factors in the Kondo regime in comparison with the results without the Coulomb interaction. Moreover, the external voltages that reduce the transmission probability evidently in the interacting QD can cause the Fano factor increasing monotonically. But in the opposite non-interaction case the Fano factor displays a profound behavior which is dependent on the energy level. In addition, due to the symmetric structure studied here, the energy-level-dependent transmission probability is exactly symmetric about the electron-hole symmetry. This is why the Fano factor for $\epsilon_{d}=-6$ coincides with that for $\epsilon_{d}=-1$. In contrast, because of the low-transparency for the non-interacting $\mathrm{QD}$ with $\epsilon_{d}=-6$, the Fano factor indicates a weak voltage-dependence and it is nearly equal to 1. In summary, we can claim that the electron-electron interaction can largely suppress the Fano factor, i.e., the shot noise of QD systems in comparison with the Poisson value.

Magnetic field is a helpful tool to probe the properties of noise. Recently, a magnetic field induced enhancement of noise is reported in the experiment 21 The theoretical investigation on noise under the influence of magnetic fields is scarce. Here we numerically study this problem in the QD. In Figs.4, we show the calculated current (a) and the zero-frequency shot noise spectrum (b) of the QD with $U=4$ and $\epsilon_{d}=-1$ versus voltages in the presence of magnetic fields from $h=0$ up to 0.5 . For the specially chosen parameters, the Kondo temperature $T_{K}$ of the QD is about 0.3. As shown in the inset figure of Fig. 4(a), the single Kondo peak in the differential conductance gradually 
splits with increasing magnetic fields and a nonzero-bias maximum appears at $h=0.5$. On the contrary, the magnetic field-dependent zero-frequency differential shot noise experiences a crossover from nonzero-bias maximum to zerobias maximum when the magnetic field increases. Again, we find that the differential shot noise demonstrates very different behavior from the differential conductance. Therefore, measuring the shot noise spectrum can improve our understanding of electric properties in QD systems.

As revealed in the experiment, 21 the magnetic fields indeed enhance the Fano factor. We can observe this behavior more clearly in Fig. 5, in which the Fano factors are illustrated as a function of voltages. This enhancement can be interpreted as the fact that the reduction of the Kondo-enhanced DOS due to applying-of magnetic fields in QD decrease the conductance or the transmission probability in the Kondo regime, 140,4 , 10 , 29 as is shown in the inset figure of Fig. 5 which depicts the magnetic-field-dependent transmission probability for the QD at several voltages. Of course, more strong magnetic fields can result in more pronounced reduction of DOS in the QD, thus can enhance the Fano factor more significantly.

\section{CONCLUSION}

We have studied the shot noise properties through QD on the basis of a new finite- $U$ SBMF approach and the nonequilibrium Green's function approach. The advantage of the present method, which the strongly interacting Hamiltonian for QD is transformed to a non-interacting one by introducing several auxiliary Boson field operators, is its capability of eliminating the crucial difficulty in calculating the current-current correlation GFs involved in the investigation of the shot noise. This renormalized Hamiltonian can make us deal with the single-particle GFs only instead of the two-particle correlation GFs. Finally, by assuming that the fluctuations of those slave-boson operators are neglected in the calculation, we derive an explicit expression for the shot noise power spectrum in terms of the Keldysh GFs in QD, which is similar with that for the non-interacting systems. The validity about this approximation and the resulting effects of including these fluctuations will be investigated in a separate publication. Generally speaking, the defined "average" shot noise spectrum $S(\omega)$ is different from the shot noise $S_{\alpha}(\omega)$ of the lead $\alpha$. Worth noting, in the limit of zero frequency the average shot noise coincides by chance with the noise of any leads at zero temperature.

In the wide band limit, the integral is performed for the zero-frequency shot noise at zero temperature. This concrete expression is utilized to analyze the Fano factors in the small voltage and large voltage limits. The Fano factor $\gamma$ is a helpful tool to measure the enhancement or suppression of the shot noise to its Poisson value. We find that the property $\gamma=1 / 2$ for symmetric systems is universal in the limit of $V \rightarrow \infty$ no matter what the strong Coulomb interaction is presence or absence and no matter what the magnetic field is applied or not.

This expression is also employed to carry out calculation for the zero-frequency shot noise power spectrum $S(0)$, zero-frequency differential shot noise $d S(0) / d V$ and the Fano factor $\gamma$ versus the external voltage at zero temperature. The numerical results show that the zero-frequency differential shot noise spectrum shows an obviously different behavior from the differential conductance, providing a beneficial information to improve understanding of electric properties in QD. Furthermore, our calculation reveals that the Fano factors are largely reduced by the Coulomb interaction, which indicates that the electron-electron interaction provides another mechanism, besides the Pauli exclusion rule, to further suppress the shot noise in QD below the Poission value.

On the other hand, the effects of magnetic fields on shot noise are also investigated numerically. An enhancement of the Fano factor is found due to the magnetic-field-induced reduction of the Kondo-enhanced DOS and the transmission probability, which is accordance with the physical expectation.

\section{ACKNOWLEDGEMENTS}

One of the authors, B. Dong, is very pleasure to acknowledge fruitful discussion with Dr. G. H. Ding. This work was supported by the National Natural Science Foundation of China, the Special Funds for Major State Basic Research Project (grant No. 2000683), the Ministry of Science and Technology of China, the Shanghai Municipal Commission of Science and Technology, and the Shanghai Foundation for Research and Development of Applied Materials. 
${ }^{1}$ D. Goldhaber-Gordon, H. Shtrikman, D. Mahalu, D. Abusch-Magder, U. Meirav and M. A. Kastner, Nature (London) 391, 156 (1998).

${ }^{2}$ S. M. Cronenwett, T. H. Oosterkamp, and L. P. Kouwenhoven, Science 281, 540 (1998).

${ }^{3}$ F. Simmel, R. H. Blick, J. P. Kotthaus, W. Wegscheider, and M. Bichler, Phys. Rev. Lett. 83, 804 (1999).

${ }^{4}$ D. C. Ralph, and R. A. Buhrman, Phys. Rev. Lett. 72, 3401 (1994).

${ }^{5}$ J. Schmid, J. Weis, K. Eberl and K. v. Klitzing, Physica B, 256-258, 182 (1998); Phys. Rev. Lett. 83, 5824 (2000).

${ }^{6}$ S. Sasaki, S. De Franceschi, J. M. Elzerman, W. G. van der Wiel, M. Eto, S. Tarucha, and L. P. Kouwenhoven, Nature 405, $764(2000)$.

${ }^{7}$ D. Goldhaber-Gordon, J. Gores, M. A. Kastner, H. Shtrikman, D. Mahalu, and U. Meirav, Phys. Rev. Lett. 81, 5225 (1998).

${ }^{8}$ W. G. van der Wiel, S. De Franceschi, T. Fujisawa, J. M. Elzerman, S. Tarucha, and L. P. Kouwenhoven, Science 289, 2105 (2000).

${ }^{9}$ Y. Meir, N. S. Wingreen, and P. A. Lee, Phys. Rev. Lett. 66, 3048 (1991).

${ }^{10}$ Y. Meir, N. S. Wingreen, P. A. Lee, Phys. Rev. Lett. 70, 2601 (1993).

${ }^{11}$ S. Hershfield, J. H. Davies, J. W. Wilkins, Phys. Rev. B 46, 7046 (1992).

${ }^{12}$ T. K. Ng, Phys. Rev. Lett. 70, 3635 (1993).

13 N. S. Wingreen and Y. Meir, Phys. Rev. B 49, 11040 (1994).

${ }^{14}$ A. L. Yeyati, A. Martín-Rodero, and F. Flores: Phys. Rev. Lett. 71, 2991 (1993).

${ }^{15}$ L. Craco and K. Kang, Phys. Rev. B 59, 12244 (1999).

${ }^{16}$ Ya. M. Blanter and M. Büttiker, Phys. Rep. 336, 1 (2000).

${ }^{17}$ M. Büttiker, Phys. Rev. Lett. 65, 2901 (1990); Phys. Rev. B 46, 12485 (1992).

${ }^{18}$ M. Reznikov, M. Heiblum, H. Shtrikman, and D. Mahalu, Phys. Rev. Lett. 75, 3340 (1995).

19 A. Kumar, L. Saminadayar, D. C. Glattli, Y. Jin, and B. Etienne, Phys. Rev. Lett. 76, 2778 (1996).

${ }^{20}$ G. Iannaccone, G. Lombardi, M. Macucci and B. Pellegrini, Phys. Rev. Lett. 80, 1054 (1998).

${ }^{21}$ V. V. Kuznetsov, E. E. Mendez, J. D. Bruno and J. T. Pham, Phys. Rev. B 58, 10159 (1998).

${ }^{22}$ K. E. Nagaev, Phys. Rev. B 57, 4628 (1998).

${ }^{23}$ M. J. M. de Jong and C. W. J. Beenakker, Phys. Rev. B 51, 16867 (1995).

${ }^{24}$ L. Y. Chen and C. S. Ting, Phys. Rev. B 43, 4534 (1991).

${ }^{25}$ Y. Wei, B. Wang, J. Wang and H. Guo, Phys. Rev. B 60, 16900 (1999).

${ }^{26}$ S. Hershfield, Phys. Rev. B 46, 7061 (1992).

${ }^{27}$ F. Yamaguchi and K. Kawamura, J. Phys. Soc. Jap. 63, 1258 (1994); Physica B 227, 116 (1996).

${ }^{28}$ G. H. Ding and T. K. Ng, Phys. Rev. B 56, 15521 (1997).

${ }^{29}$ B. Dong and X. L. Lei, Phys. Rev B 63, 235306 (2001); B. Dong and X. L. Lei, J. Phys.: Cond. Matter 13, 9245 (2001).

${ }^{30}$ G. Kotliar, and A. E. Ruckenstein, Phys. Rev. Lett. 57, 1362 (1986).

${ }^{31}$ D. C. Langreth, in Linear and Nonlinear Electron Transport in Solids, Nato ASI, Series B vol. 17, Ed. J. T. Devreese and V. E. Van Doren (Plenum, New York, 1976).

32 T. K. Ng and P. A. Lee, Phys. Rev. Lett. 61, 1768 (1988).

${ }^{33}$ U. Gerland, J. vonDelft, T. A. Costi, and Y. Oreg, Phys. Rev. Lett. 84, 3710 (2000).

\section{FIGURE CAPTIONS}

Fig. 1 (a) The current $I$, (b) the zero-frequency shot noise power spectrum $S(0)$, (c) the differential conductance $d I / d V$, and (d) the zero-frequency differential shot noise spectrum $d S(0) / d V$, at zero temperature, as a function of voltage for the QD energy levels $\epsilon_{d}=-1,-1.5,-2,-3.5$, and -6 with the on-site Coulomb interaction $U=7$.

Fig. 2 The zero-frequency shot noise power spectrum $S(0)$ at zero temperature as a function of voltage for the QD with $U=0$ and the same energy levels as Fig. 1. The inset figure: the corresponding zero-frequency differential shot noise.

Fig. 3 (a) The Fano factor vs voltage for the QD with $U=7$. (b) The Fano factor vs voltage for the QD with $U=0$. The other parameters are the same as in Fig. 1. (c) The transmission coefficient vs the energy level of the QD with $U=7$ (thick lines) and $U=0$ (thin lines) at several voltages $V=0,0.1,0.2$, and 0.5 ( $U=0$ only).

Fig. 4 (a) The current and (b) the zero-frequency shot noise power spectrum at zero temperature vs voltage for the QD with the energy level $\epsilon_{d}=-1$ and with the on-site Coulomb interaction $U=4$ in different applied magnetic fields $h=0,0.1,0.2,0.3,0.4$, and 0.5 . Inset: (a) the differential conductance vs voltage; (b) the zero-frequency differential shot noise vs voltage.

Fig. 5 The Fano factor vs voltage for the QD with the same parameters as in Fig. 4. Inset: The transmission coefficient vs the magnetic field at different voltages $V=0,0.2$, and 0.3 . 


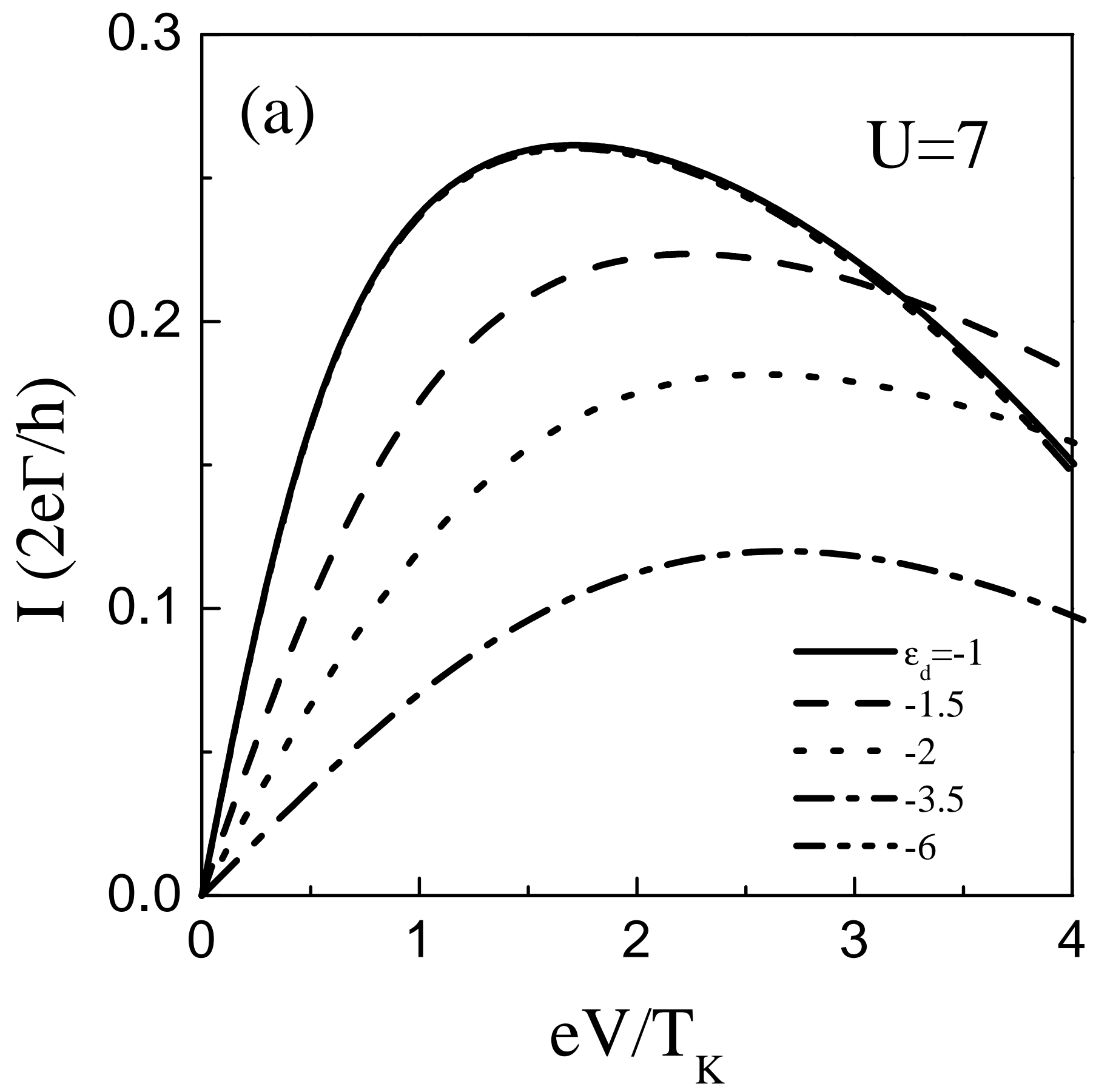




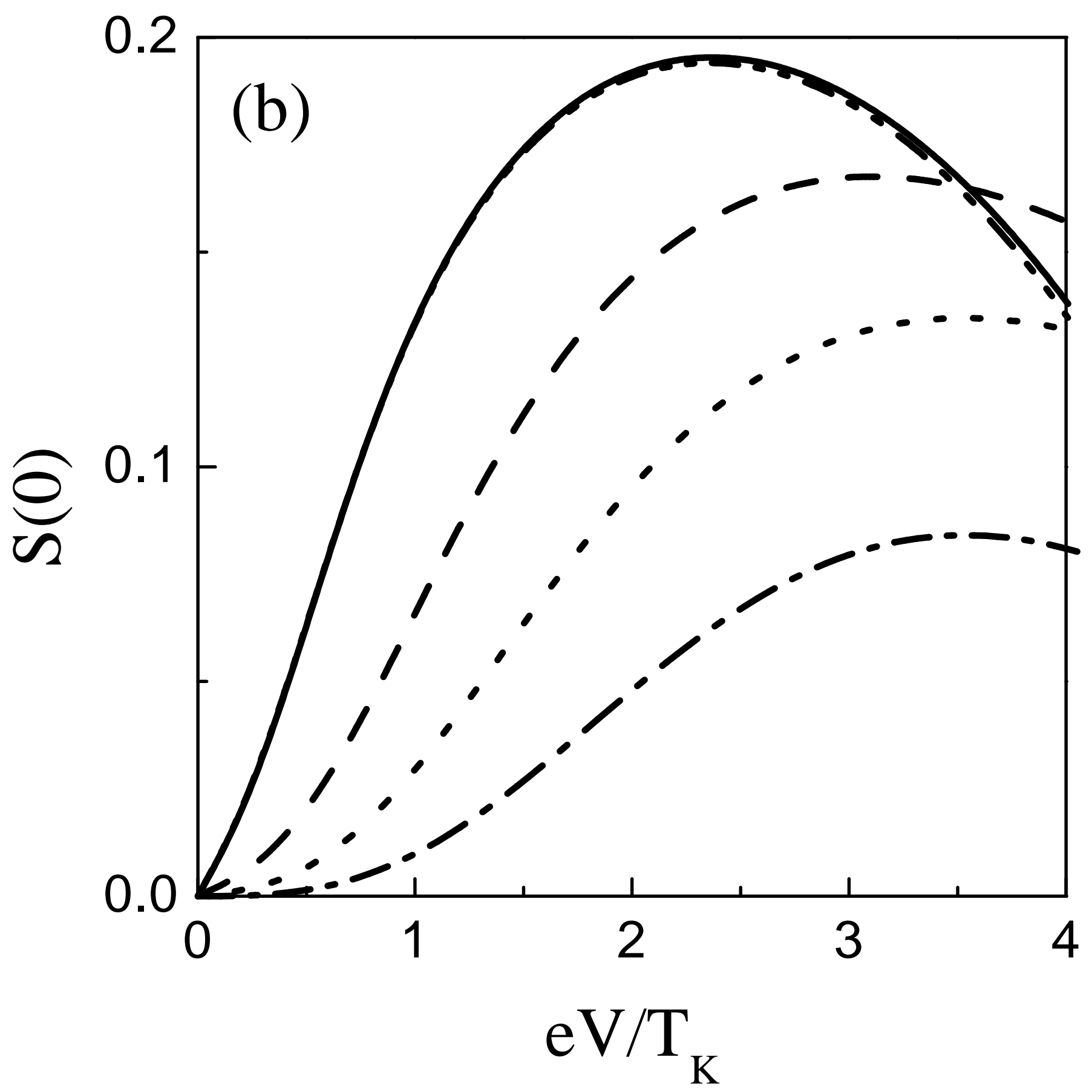




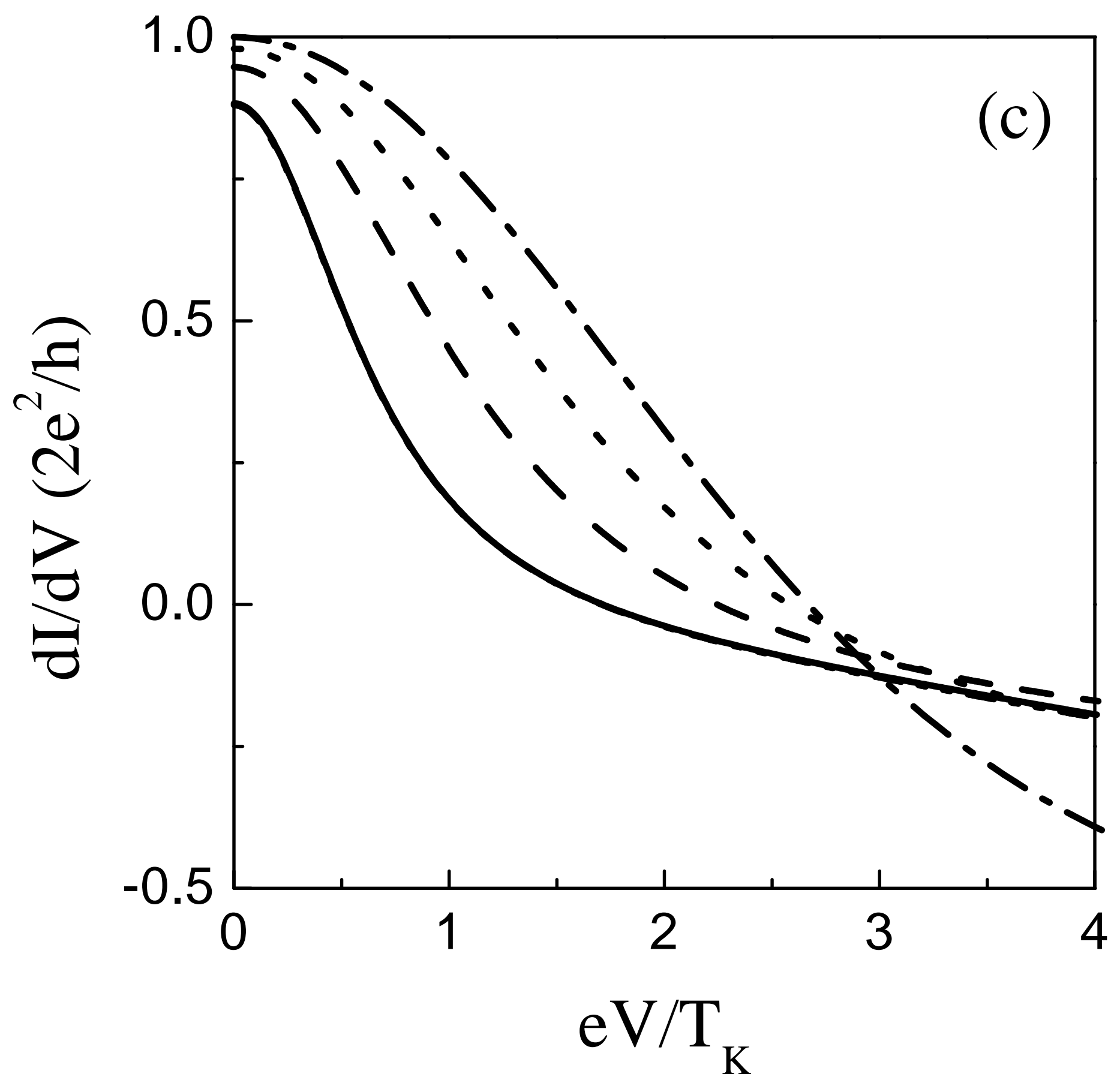




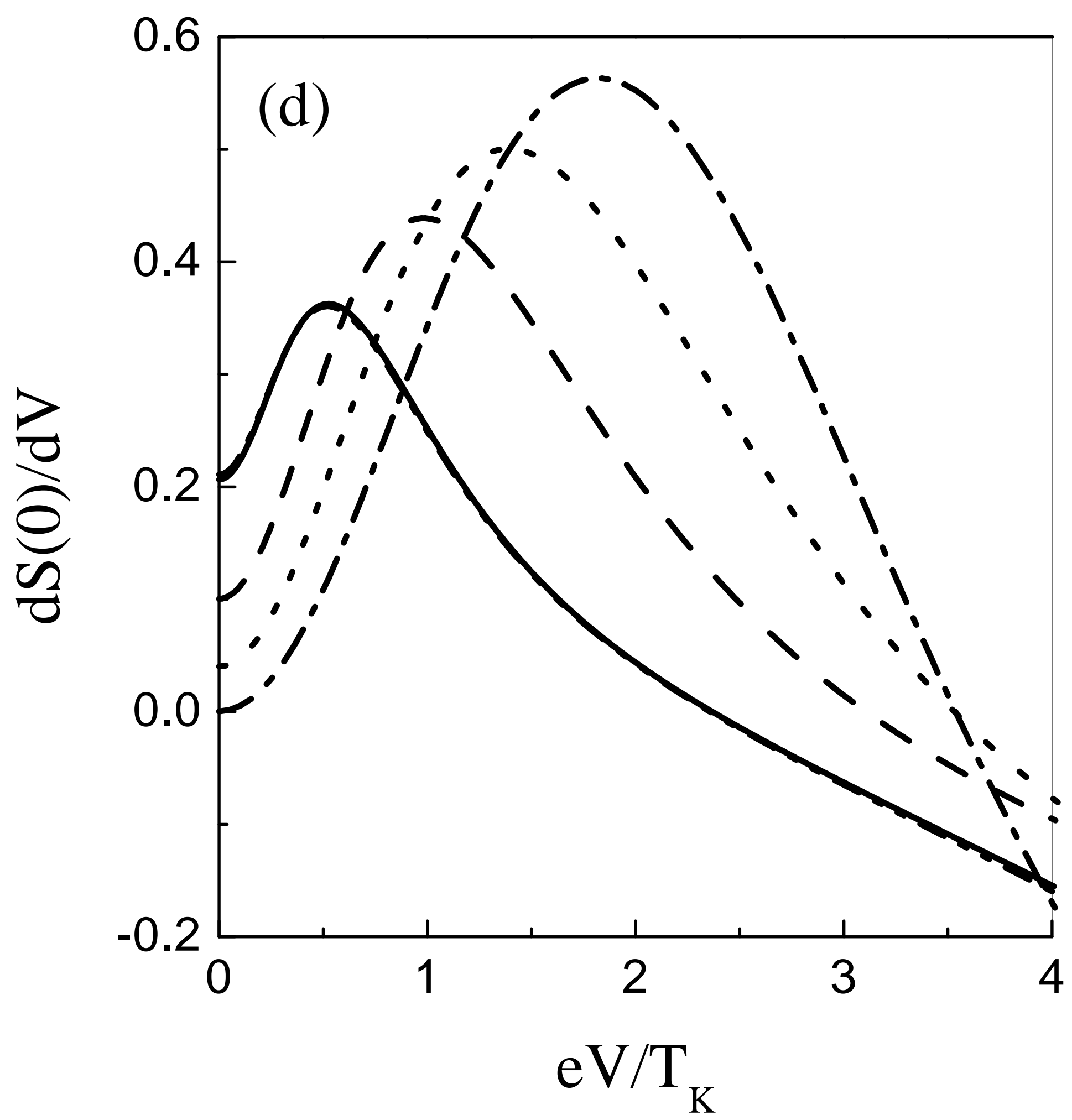




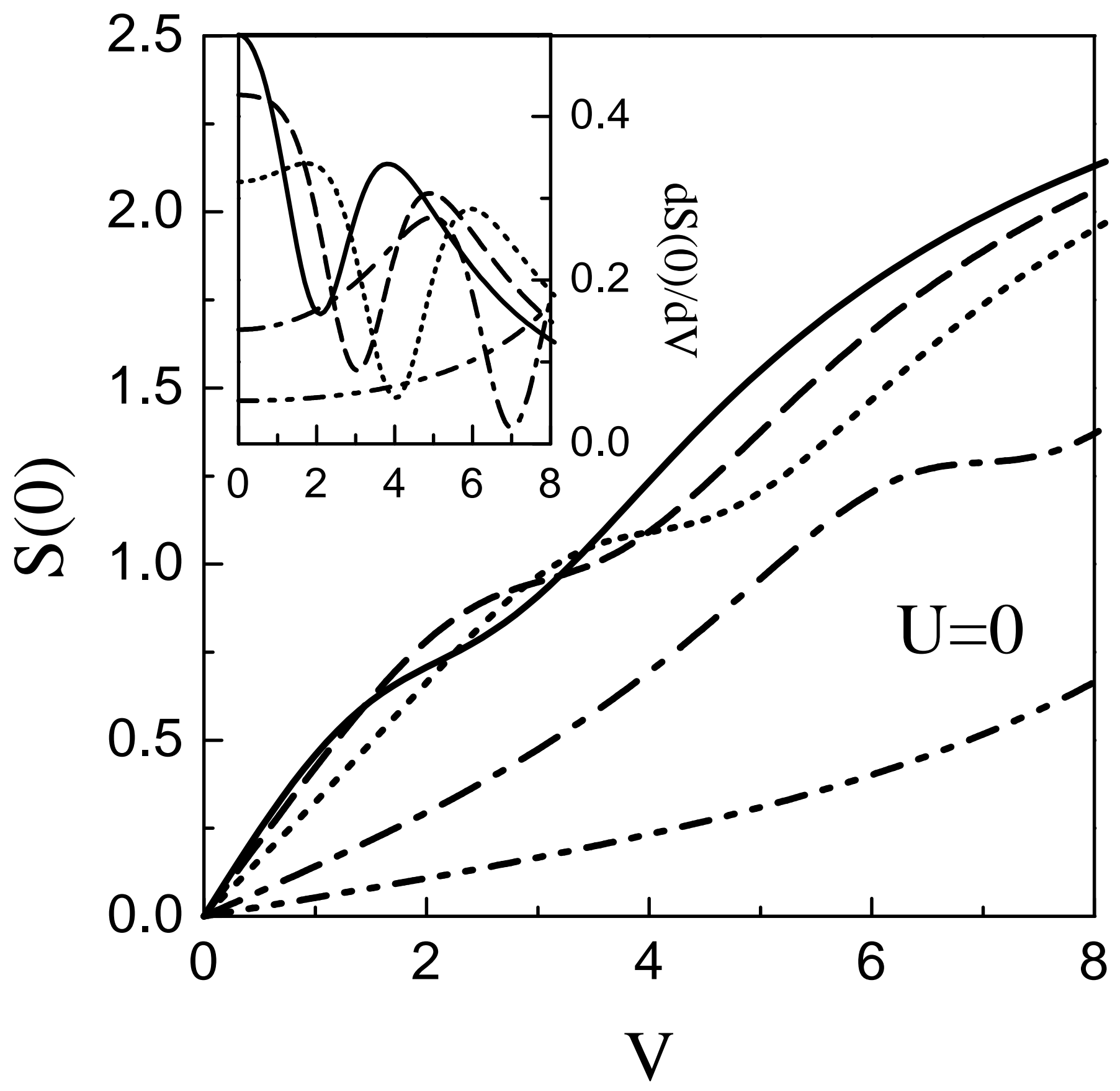




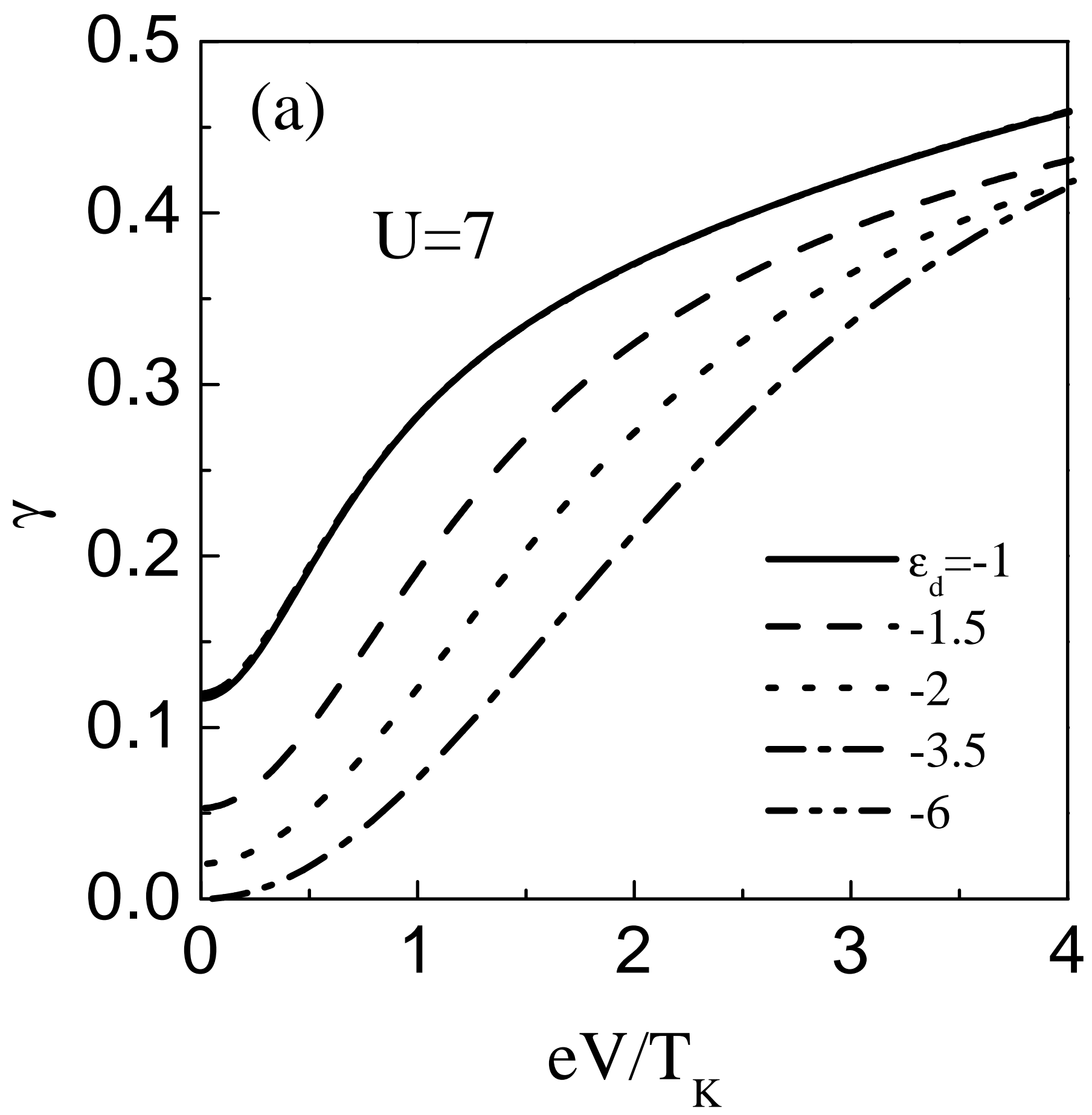




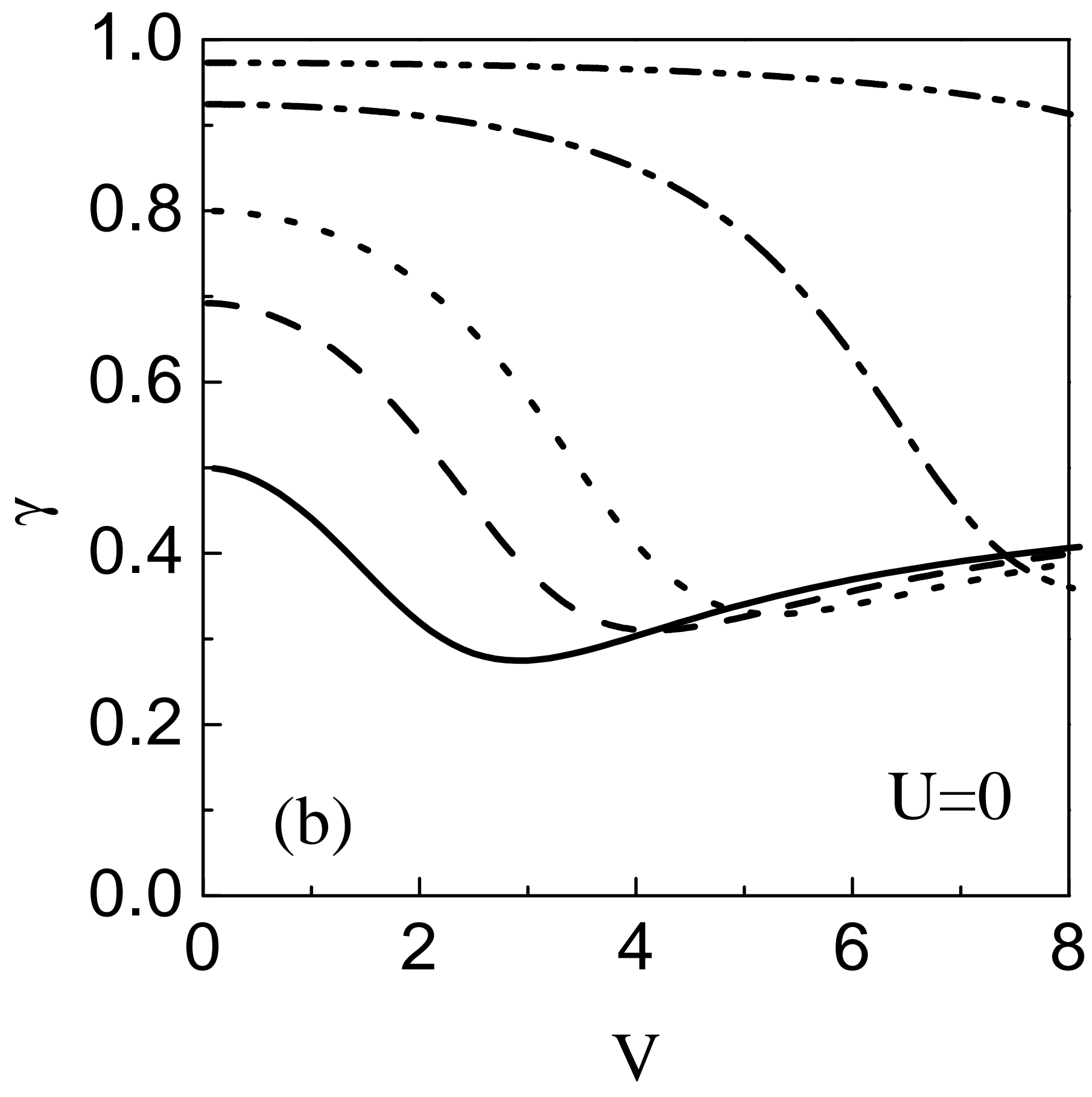




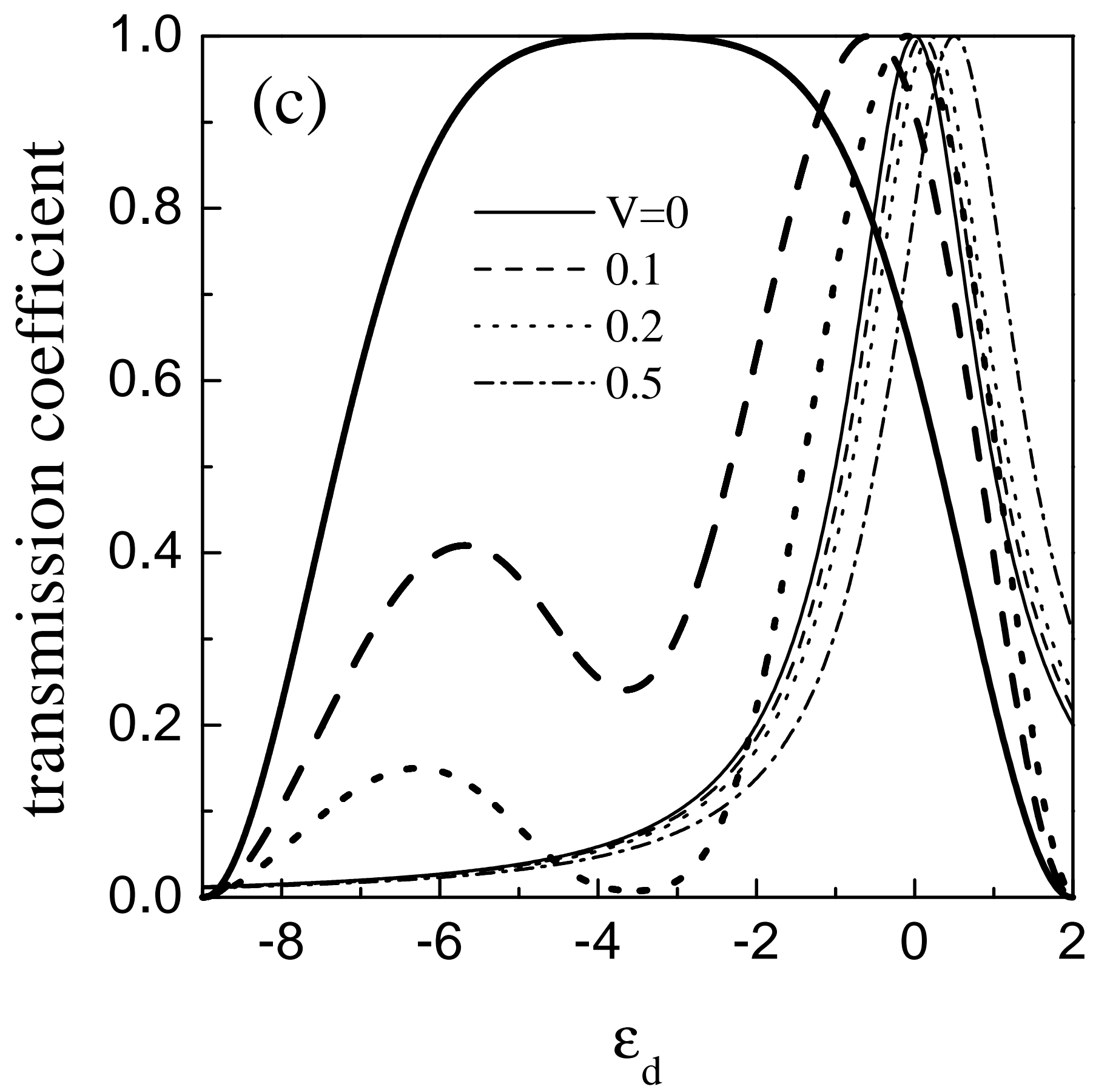




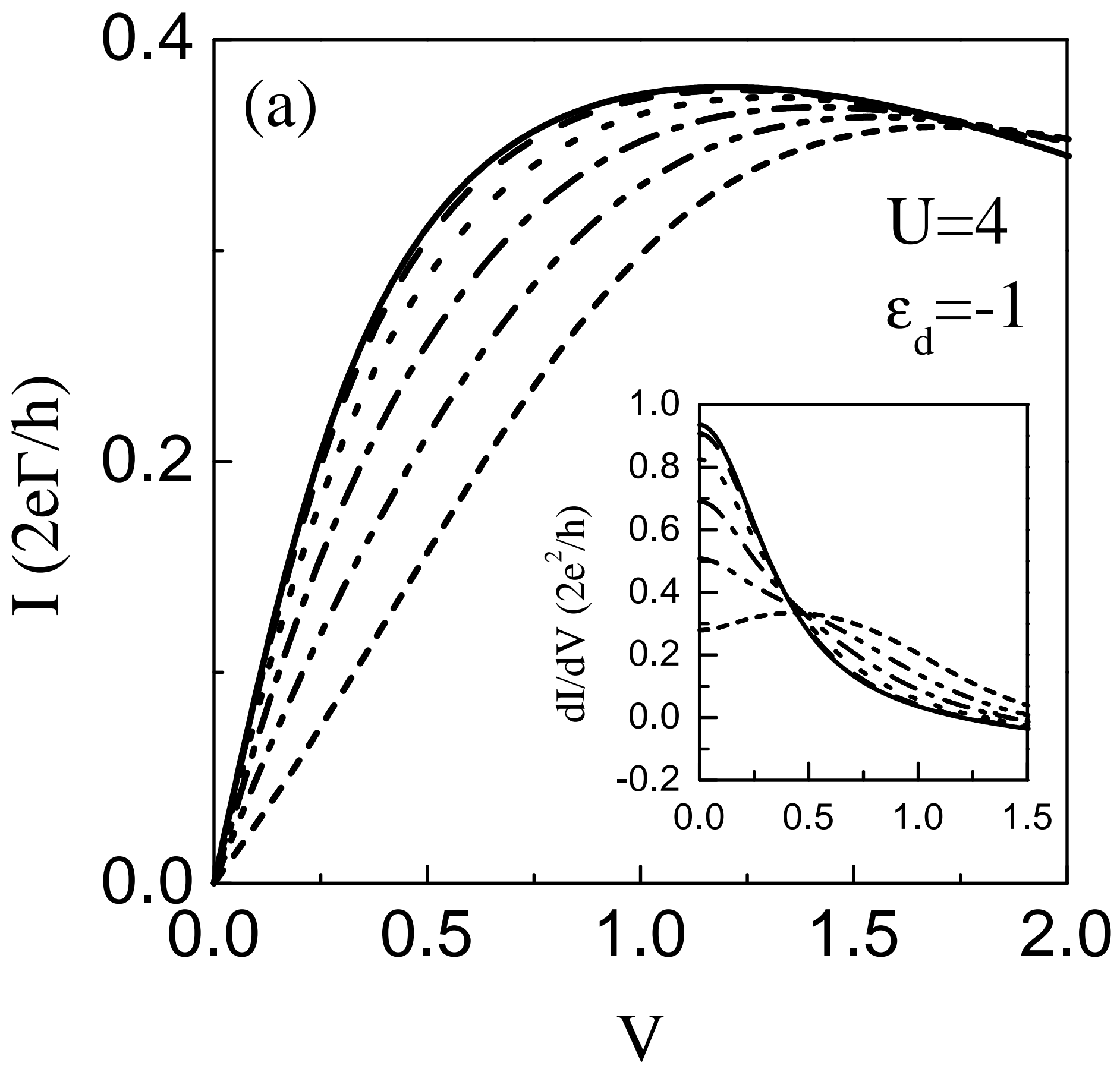




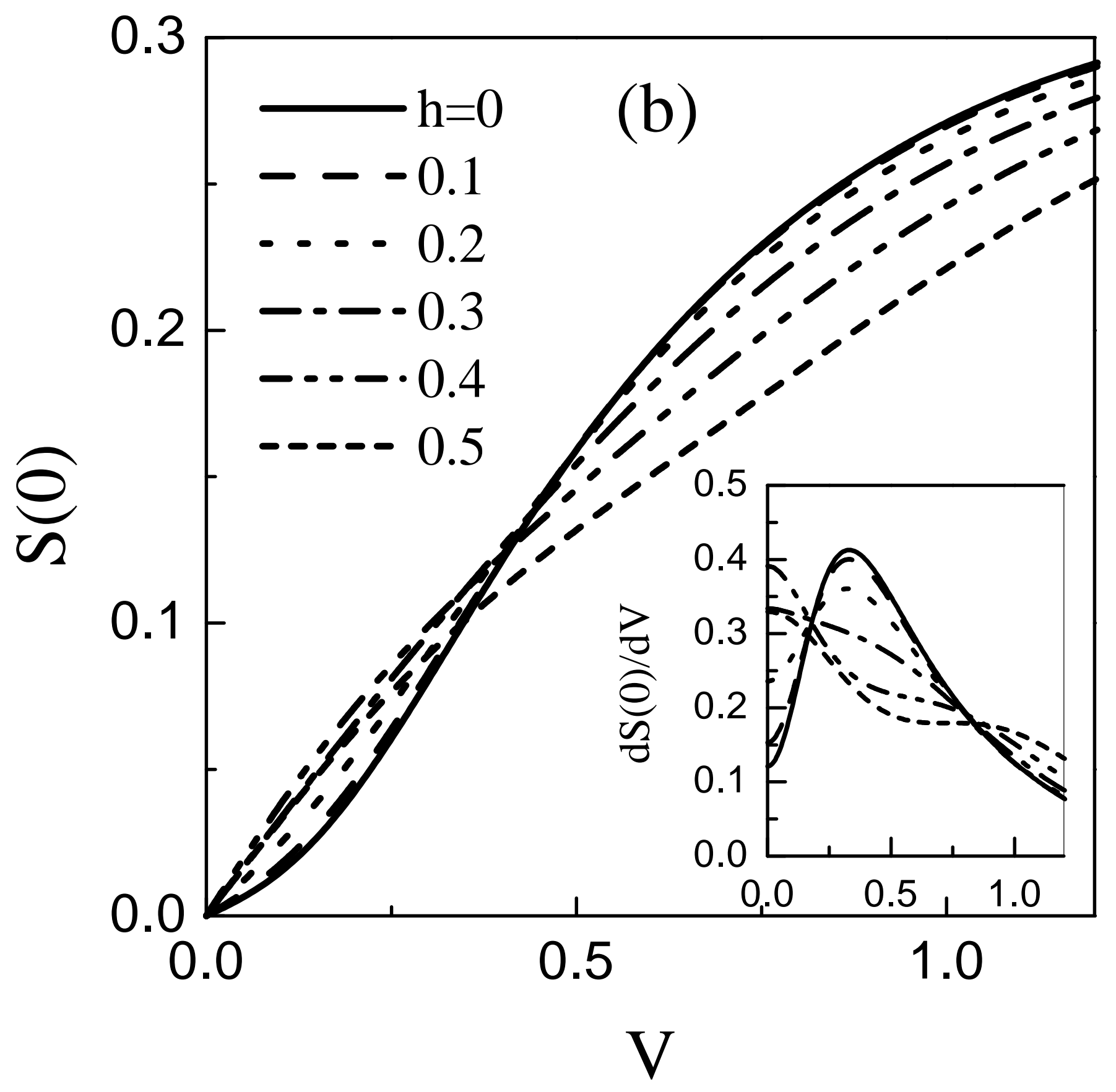




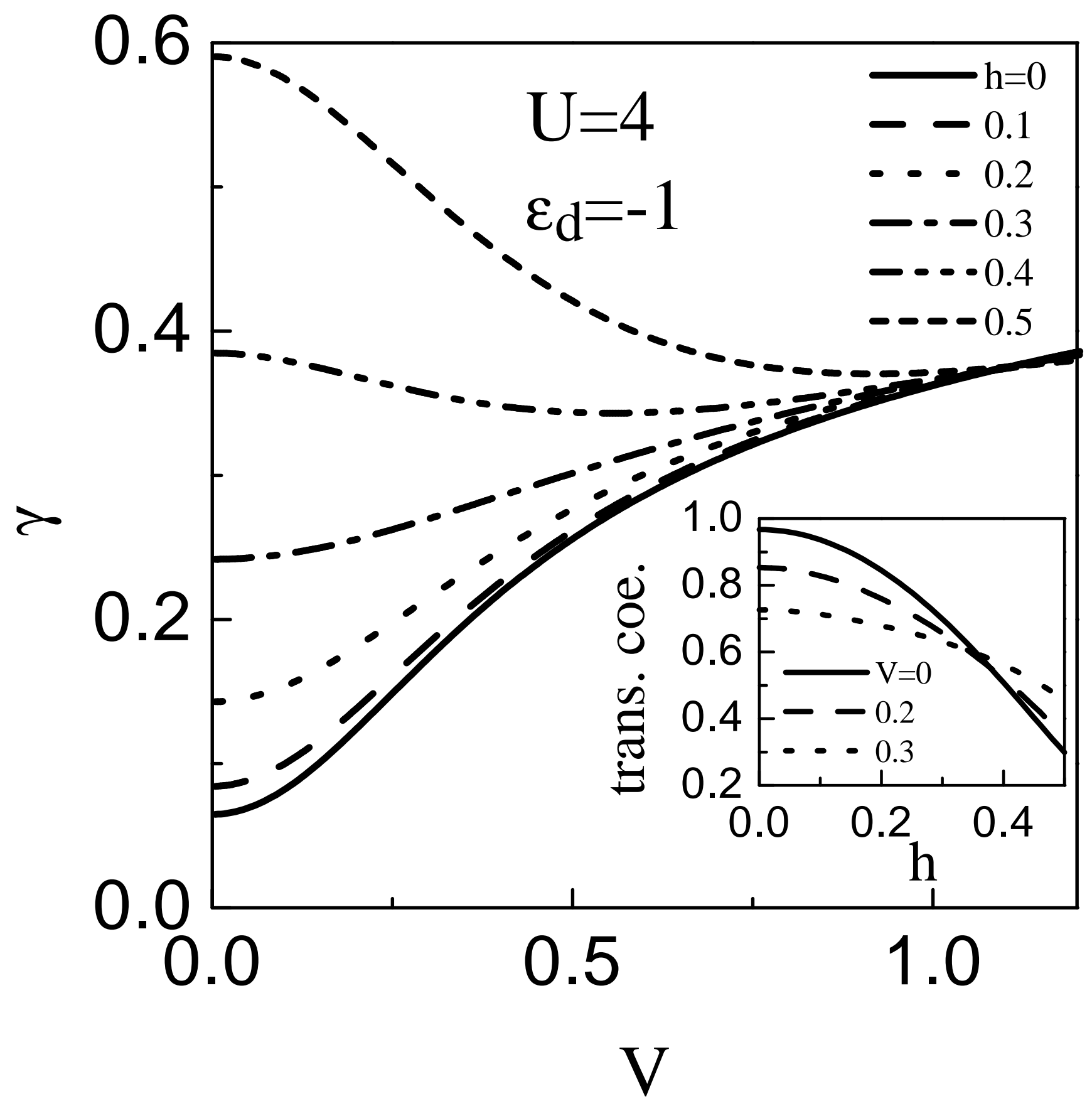

